\title{
Some operational properties of the Laguerre transform
}

M. M. Rodrigues, V. N. Huy, and N. M. Tuan

Citation: 1798, 020130 (2017); doi: 10.1063/1.4972722

View online: http://dx.doi.org/10.1063/1.4972722

View Table of Contents: http://aip.scitation.org/toc/apc/1798/1

Published by the American Institute of Physics 


\title{
Some operational properties of the Laguerre transform
}

\author{
M. M. Rodrigues, ${ }^{1, a)}$, V.N. Huy ${ }^{2, b)}$ and N.M. Tuan ${ }^{3, c)}$ \\ ${ }^{1}$ CIDMA - Center for Research and Development in Mathematics and Applications Department of Mathematics, \\ University of Aveiro Campus Universitário de Santiago, 3810-193 Aveiro, Portugal. \\ ${ }^{2}$ Department of Mathematics, College of Science, Vietnam National University, 334 Nguyen Trai, Thanh Xuan, \\ Hanoi, Vietnam. \\ ${ }^{3}$ Dept. of Mathematics, College of Education, Vietnam National University, G7 Build, 144 Xuan Thuy Rd., Cau Giay, \\ Dist., Hanoi, Vietnam.

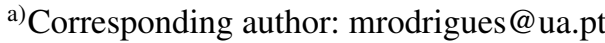 \\ b) nhat-huy85@yahoo.com \\ c)nguyentuan@vnu.edu.vn
}

\begin{abstract}
This paper is devoted to the study of some properties of the Laguerre transform. We define new properties of the Laguerre transform in a weighted $L_{2}$-space. Moreover, we present some results concerning the action of this integral transform over some class of polynomials.
\end{abstract}

\section{INTRODUCTION}

The Laguerre polynomials appear naturally in many branches of pure and applied mathematics and mathematical physics (see e.g. [1, 2, 3, 6]). Debnath [1] introduced the Laguerre transform and derived some of its properties. He also discussed the applications in study of heat conduction [3] and to the oscillations of a very long and heavy chain with variable tension [2]. Moreover, application of the integral Laguerre transforms for forward seismic modeling can be seen in [5].

The Laguerre transform of a function $f(x)$ is denoted by $\tilde{f}_{\alpha}(n)$ and defined by the integral

$$
L\{f(x)\}=\widetilde{f}_{\alpha}(n)=\int_{0}^{\infty} e^{-x} x^{\alpha} L_{n}^{\alpha}(x) f(x) d x, n=0,1,2, \ldots
$$

provided the integral exists in the sense of Lesbegue, where $L_{n}^{\alpha}(x)$ is a generalized Laguerre polynomial of degree $n$ with order $\alpha>-1$, and satisfies the following differential equation

$$
\frac{d}{d x}\left[e^{-x} x^{\alpha+1} \frac{d}{d x} L_{n}^{\alpha}(x)\right]+n e^{-x} x^{\alpha} L_{n}^{\alpha}(x)=0 .
$$

The sequence of Laguerre polynomial $\left(L_{n}^{\alpha}(x)\right)_{n=0}^{\infty}$ have the following property:

$$
\int_{0}^{\infty} e^{-x} x^{\alpha} L_{n}^{\alpha}(x) L_{m}^{\alpha}(x) d x=\left(\begin{array}{l}
n+\alpha \\
n
\end{array}\right) \Gamma(\alpha+1) \delta_{n m},
$$

where $\delta_{n m}$ is Kronecker function defined by

$$
\delta_{n m}=\left\{\begin{array}{l}
1, \text { if } n=m \\
0, \text { if } n \neq m
\end{array}\right.
$$

and $\Gamma(\alpha+1)=\int_{0}^{\infty} x^{\alpha} e^{-x} d x$ 
The inverse of the Laguerre transformation is then

$$
f(x)=\sum_{n=0}^{\infty}\left(\delta_{n}\right)^{-1} \widetilde{f}_{\alpha}(n) L_{n}^{\alpha}(x)(0<x<\infty),
$$

where $\delta_{n}=\left(\begin{array}{c}n+\alpha \\ n\end{array}\right) \Gamma(\alpha+1)$.

This paper is devoted to the study of the generalized Laguerre transform and some operational properties.

\section{MAIN RESULTS}

In this section we define new properties for the Laguerre transform in a weighted $L_{2}-$ space. Moreover, we present some results concerning the action of this integral transform over some class of polynomial.

For $1 \leq p \leq \infty$ the space $L_{p, \alpha}$ is defined via the following formula

$$
L_{p, \alpha}=\left\{f:(0, \infty) \rightarrow \mathbb{R}: \quad \int_{0}^{\infty}|f(x)|^{p} e^{-x} x^{\alpha} d x<\infty\right\}
$$

with the norm

$$
\|f\|_{L_{p, \alpha}}=\left(\int_{0}^{\infty}|f(x)|^{p} e^{-x} x^{\alpha} d x\right)^{1 / p}
$$

where the convention that

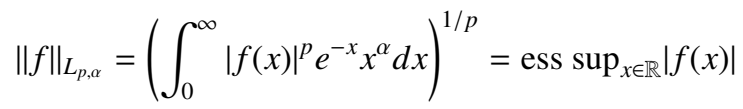

if $p=\infty$.

Clearly, if $f$ is an arbitrary polynomial then $f \in L_{p, \alpha}$. We also define

$$
l_{p, \alpha}=\left\{f=(f(n))_{n=0}^{\infty}: \sum_{n=0}^{\infty}|f(n)|^{p}\left(\begin{array}{c}
n+\alpha \\
n
\end{array}\right)<\infty\right\}
$$

with the norm

$$
\|f\|_{l_{p, \alpha}}=\left(\sum_{n=0}^{\infty}|f(n)|^{p}\left(\begin{array}{c}
n+\alpha \\
n
\end{array}\right)\right)^{1 / p}
$$

We define the differential operator $R$ via the formula

$$
\begin{aligned}
R[f(x)] & =e^{x} x^{-\alpha} \frac{d}{d x}\left[e^{-x} x^{\alpha+1} \frac{d}{d x} f(x)\right] \\
& =x f^{\prime \prime}(x)+(\alpha+1-x) f^{\prime}(x)
\end{aligned}
$$

and then for $n=0,1, \ldots$, we have

$$
R^{n}[f(x)]=R\left[R^{n-1}[f(x)]\right]
$$

Let $P(x)$ be a polynomial. The differential operator $P(R)$ is obtain from $P(x)$ by substituting $x \rightarrow R$. i.e., if $P(x)=\sum_{k=0}^{n} a_{k} x^{k}$ then

$$
P(R)[f(x)]=\sum_{k=0}^{n} a_{k} R^{k}[f(x)]
$$

Moreover, we denote by

$$
\operatorname{supp} \widetilde{f_{\alpha}}=\left\{n \in \mathbb{Z}_{+}: \widetilde{f}_{\alpha}(n) \neq 0\right\}
$$

the support of the Laguerre transform of $f$.

Now, we have the following theorem 
Theorem $0.1 \quad$ Let $1 \leq p<\infty, P(x)$ be a polynomial. Then for an arbitrary infinitely differentiable function $f \in L_{p, \alpha}$, there exist the following limit

$$
\lim _{m \rightarrow \infty}\left\|P^{m}(R)[f(x)]\right\|_{L_{p, \alpha}}^{1 / m}
$$

and

$$
\lim _{m \rightarrow \infty}\left\|P^{m}(R)[f(x)]\right\|_{L_{p, \alpha}}^{1 / m}=\sup \left\{|P(-n)|: n \in \operatorname{supp} \widetilde{f}_{\alpha}\right\} .
$$

Proof: From the definition of differential operator $R$, we get

$$
\begin{gathered}
L\{R[f(x)]\}=\int_{0}^{\infty} e^{-x} x^{\alpha} L_{n}^{\alpha}(x) e^{x} x^{-\alpha} \frac{d}{d x}\left[e^{-x} x^{\alpha+1} \frac{d f}{d x}\right] d x \\
=\int_{0}^{\infty} L_{n}^{\alpha}(x) \frac{d}{d x}\left[e^{-x} x^{\alpha+1} \frac{d f}{d x}\right] d x .
\end{gathered}
$$

Using the integral by part, we have

$$
\begin{aligned}
L\{R[f(x)]\} & =\left[e^{-x} x^{\alpha+1} L_{n}^{\alpha}(x) \frac{d f}{d x}\right]_{0}^{\infty}-\int_{0}^{\infty} e^{-x} x^{\alpha+1} \frac{d L_{n}^{\alpha}(x)}{d x} \frac{d f}{d x} d x \\
& =-\int_{0}^{\infty} e^{-x} x^{\alpha+1} \frac{d L_{n}^{\alpha}(x)}{d x} \frac{d f}{d x} d x .
\end{aligned}
$$

We see that

$$
\begin{aligned}
-\int_{0}^{\infty} e^{-x} x^{\alpha+1} \frac{d L_{n}^{\alpha}(x)}{d x} \frac{d f}{d x} d x= & {\left[e^{-x} x^{\alpha+1} \frac{d L_{n}^{\alpha}(x)}{d x} f(x)\right]_{0}^{\infty} } \\
& +\int_{0}^{\infty} \frac{d}{d x}\left[e^{-x} x^{\alpha+1} \frac{d L_{n}^{\alpha}(x)}{d x}\right] f(x) d x \\
& =\int_{0}^{\infty} \frac{d}{d x}\left[e^{-x} x^{\alpha+1} \frac{d L_{n}^{\alpha}(x)}{d x}\right] f(x) d x .
\end{aligned}
$$

Then, from (0.2) it follows

$$
-\int_{0}^{\infty} e^{-x} x^{\alpha+1} \frac{d L_{n}^{\alpha}(x)}{d x} \frac{d f}{d x} d x=-n \int_{0}^{\infty} e^{-x} x^{\alpha} f(x) L_{n}^{\alpha}(x) d x .
$$

Hence

$$
L\{R[f(x)]\}=-n \widetilde{f_{\alpha}}(n) .
$$

Similarly, we get

$$
L\left\{R^{m}[f(x)]\right\}=(-1)^{m} n^{m} \widetilde{f}_{\alpha}(n) .
$$

Hence, from this and the definition of $P^{m}(R)$ we obtain

$$
L\left\{P^{m}(R)[f(x)]\right\}=P^{m}(-n) \widetilde{f_{\alpha}}(n) .
$$

Now, let consider $\sigma$ an arbitrary number in $\operatorname{supp} \widetilde{f_{\alpha}}$. Then

$$
\int_{0}^{\infty} e^{-x} x^{\alpha} L_{\sigma}^{\alpha}(x) P^{m}(R)[f(x)] d x=R^{m}(-\sigma) \widetilde{f}_{\alpha}(\sigma) .
$$

Applying Hölder inequality, we obtain

$$
\begin{aligned}
\left|\int_{0}^{\infty} e^{-x} x^{\alpha} L_{\sigma}^{\alpha}(x) P^{m}(R)[f(x)] d x\right| \leq & \left(\int_{0}^{\infty} e^{-x} x^{\alpha}\left|L_{\sigma}^{\alpha}(x)\right|^{q} d x\right)^{1 / q} \\
& \times\left(\int_{0}^{\infty} e^{-x} x^{\alpha}\left|P^{m}(R)[f(x)]\right|^{p} d x\right)^{1 / p} .
\end{aligned}
$$


Therefore, it follows from (0.10) and (0.11) that

$$
\left|P^{m}(-\sigma) \widetilde{f}_{\alpha}(\sigma)\right| \leq\left(\int_{0}^{\infty} e^{-x} x^{\alpha}\left|L_{\sigma}^{\alpha}(x)\right|^{q} d x\right)^{1 / q} \int_{0}^{\infty} e^{-x} x^{\alpha}\left|P^{m}(R)[f(x)]\right|^{p} d x .
$$

That mean

$$
\left|P^{m}(-\sigma) \widetilde{f}_{\alpha}(\sigma)\right| \leq\left(\int_{0}^{\infty} e^{-x} x^{\alpha}\left|L_{\sigma}^{\alpha}(x)\right|^{q} d x\right)^{1 / q}\left\|P^{m}(R)[f(x)]\right\|_{L_{p, \alpha}} .
$$

As $L_{\sigma}^{\alpha}$ is a polynomial, we obtain $L_{\sigma}^{\alpha} \in L_{q, \alpha}$ and then

$$
\left(\int_{0}^{\infty} e^{-x} x^{\alpha}\left|L_{\sigma}^{\alpha}(x)\right|^{q} d x\right)^{1 / q}<\infty .
$$

It follows from $\widetilde{f}_{\alpha}(\sigma) \neq 0$ and $(0.13)$ that

$$
|P(-\sigma)| \leq \underline{\lim }_{m \rightarrow \infty}\left\|P^{m}(R)[f(x)]\right\|_{L_{p, \alpha}}^{1 / m} .
$$

Since (0.14) holds for all $\sigma \in \operatorname{supp} \widetilde{f_{\alpha}}$, we obtain

$$
\sup \left\{|P(-\sigma)|: \quad \sigma \in \operatorname{supp} \widetilde{f}_{\alpha}\right\} \leq \underline{\lim }_{m \rightarrow \infty}\left\|P^{m}(R)[f(x)]\right\|_{L_{p, \alpha}}^{1 / m} .
$$

Now, we will prove that

$$
\sup \left\{|P(-\sigma)|: \quad \sigma \in \operatorname{supp} \widetilde{f}_{\alpha}\right\} \geq \varlimsup_{\lim _{m \rightarrow \infty}}\left\|P^{m}(R)[f(x)]\right\|_{L_{p, \alpha}}^{1 / m} .
$$

Indeed, if supp $\widetilde{f_{\alpha}}$ is an unbounded set then

$$
\sup \left\{|P(-\sigma)|: \sigma \in \operatorname{supp} \widetilde{f}_{\alpha}\right\}=\infty
$$

and (0.16) holds. Now, we only need to prove $(0.16)$ for the case that $\operatorname{supp} \widetilde{f}_{\alpha}$ is a bounded set, that mean, $f$ is a polynomial and

$$
M:=\sup \left\{|n|: n \in \operatorname{supp} \widetilde{f}_{\alpha}\right\}<\infty .
$$

Hence

$$
P^{m}(R)[f(x)]=\sum_{n=-M}^{M}\left(\delta_{n}\right)^{-1} P^{m}(-n) \widetilde{f}_{\alpha}(n) L_{n}^{\alpha}(x)(0<x<\infty)
$$

and

$$
\begin{gathered}
\quad \leq \sum_{n=-M}^{M}\left(\delta_{n}\right)^{-1} P^{m}(-\sigma)[f(x)]\left\|_{L_{p, \alpha}}(n)\right\| L_{n}^{\alpha} \|_{L_{p, \alpha}} \\
\leq\left[\sup \left\{|P(-\sigma)|: n \in \operatorname{supp} \widetilde{f}_{\alpha}\right\}\right]^{m} \sum_{n=-M}^{M}\left(\delta_{n}\right)^{-1} \widetilde{f}_{\alpha}(n)\left\|L_{n}^{\alpha}\right\|_{L_{p, \alpha}}
\end{gathered}
$$

which gives

$$
\varlimsup_{m \rightarrow \infty}\left\|P^{m}(R)[f(x)]\right\|_{L_{p, \alpha}}^{1 / m} \leq \sup \left\{|P(-\sigma)|: \quad \sigma \in \operatorname{supp} \widetilde{f}_{\alpha}\right\}
$$

Therefore, (0.16) have been proved. From $(0.15)$ and $(0.16)$, it is easy to see

$$
\lim _{m \rightarrow \infty}\left\|P^{m}(R)[f(x)]\right\|_{L_{p, \alpha}}^{1 / m}=\sup \left\{|P(-n)|: n \in \operatorname{supp} \widetilde{f_{\alpha}}\right\}
$$

and then the proof is complete.

Let $P(x)=x$. Then, according Theorem 0.1 we obtain 
Corollary $0.2 \quad$ Let $1 \leq p \leq \infty, P(x)$ be a polynomial. Then for an arbitrary arbitrary infinitely differentiable function $f \in L_{p, \alpha}$, there exist the following limit

$$
\lim _{m \rightarrow \infty}\left\|R^{m}[f(x)]\right\|_{L_{p, \alpha}}^{1 / m}
$$

and

$$
\lim _{m \rightarrow \infty}\left\|R^{m}[f(x)]\right\|_{L_{p, \alpha}}^{1 / m}=\operatorname{deg}(f) .
$$

Now, let consider an infinitely differentiable function $f:(0, \infty) \rightarrow \mathbb{R}$. Assume that we can define the sequence of function $\left(S^{m}[f(x)]\right)_{m=1}^{\infty}$ satisfying the condition

$$
R\left(S^{m}\right)[f(x)]=S^{m-1}[f(x)], \forall m \in \mathbb{N} .
$$

We also see that, for an arbitrary polynomial $f$ we get $\widetilde{f}_{\alpha}(0)=0$ if and only if the sequence of function $\left(S^{m}[f(x)]\right)_{m=1}^{\infty}$ is well defined and

$$
S^{m}[f(x)]=\sum_{n=1}^{\infty}\left(\delta_{n}\right)^{-1}\left(\frac{-1}{n}\right)^{m} \widetilde{f}_{\alpha}(n) L_{n}^{\alpha}(x) .
$$

Next, we state the following theorem

Theorem 0.3 Let consider $P(x)$ a polynomial and $1 \leq p \leq \infty$. Then for an arbitrary infinitely differentiable function $f \in L_{p, \alpha}$ satisfying $\widetilde{f}_{\alpha}(0)=0$, we have

$$
\lim _{m \rightarrow \infty}\left\|P^{m}(S)[f(x)]\right\|_{L_{2, \alpha}}^{1 / m} \geq \sup \left\{\left|P\left(\frac{-1}{n}\right)\right|: n \in \operatorname{supp} \widetilde{f}_{\alpha}\right\} .
$$

Moreover, if $f$ is a polynomial then

$$
\lim _{m \rightarrow \infty}\left\|P^{m}(S)[f(x)]\right\|_{L_{2, \alpha}}^{1 / m}=\sup \left\{\left|P\left(\frac{-1}{n}\right)\right|: n \in \operatorname{supp} \widetilde{f}_{\alpha}\right\} .
$$

Proof: We have known that

$$
L\{f(x)\}=L\{R(S)[f(x)]\}=n L\{S[f(x)]\} .
$$

We deduce that

$$
L\{S[f(x)]\}=\frac{-1}{n} \widetilde{f}_{\alpha}(n) .
$$

Similarly, we get

$$
L\left\{S^{m}[f(x)]\right\}=\left(\frac{-1}{n}\right)^{m} \widetilde{f}_{\alpha}(n) .
$$

From this and the definition of $P^{m}(S)$, we obtain

$$
L\left\{P^{m}(S)[f(x)]\right\}=P^{m}\left(\frac{-1}{n}\right) \widetilde{f}_{\alpha}(n) .
$$

Now, we consider $\sigma$ an arbitrary number in $\operatorname{supp} \widetilde{f}_{\alpha}$. Then

$$
\int_{0}^{\infty} e^{-x} x^{\alpha} L_{\sigma}^{\alpha}(x) P^{m}(S)[f(x)] d x=R^{m}\left(\frac{-1}{\sigma}\right) \widetilde{f}_{\alpha}(\sigma) .
$$

Applying Hölder inequality we obtain

$$
\begin{aligned}
\left|\int_{0}^{\infty} e^{-x} x^{\alpha} L_{\sigma}^{\alpha}(x) P^{m}(S)[f(x)] d x\right| \leq & \left(\int_{0}^{\infty} e^{-x} x^{\alpha}\left|L_{\sigma}^{\alpha}(x)\right|^{q} d x\right)^{1 / q} \\
& \times\left(\int_{0}^{\infty} e^{-x} x^{\alpha}\left|P^{m}(S)[f(x)]\right|^{p} d x\right)^{1 / p} .
\end{aligned}
$$


Therefore, it follows from (0.23) and (0.24) that

$$
\left|P^{m}\left(\frac{-1}{\sigma}\right) \widetilde{f}_{\alpha}(\sigma)\right| \leq\left(\int_{0}^{\infty} e^{-x} x^{\alpha}\left|L_{\sigma}^{\alpha}(x)\right|^{q} d x\right)^{1 / q} \int_{0}^{\infty} e^{-x} x^{\alpha}\left|P^{m}(S)[f(x)]\right|^{p} d x .
$$

That mean

$$
\left|P^{m}\left(\frac{-1}{\sigma}\right) \widetilde{f}_{\alpha}(\sigma)\right| \leq\left(\int_{0}^{\infty} e^{-x} x^{\alpha}\left|L_{\sigma}^{\alpha}(x)\right|^{q} d x\right)^{1 / q}\left\|P^{m}(S)[f(x)]\right\|_{L_{p, \alpha}} .
$$

As $L_{\sigma}^{\alpha}$ is a polynomial, we obtain $L_{\sigma}^{\alpha} \in L_{q, \alpha}$ and then

$$
\left(\int_{0}^{\infty} e^{-x} x^{\alpha}\left|L_{\sigma}^{\alpha}(x)\right|^{q} d x\right)^{1 / q}<\infty
$$

Then it follows from $\widetilde{f}_{\alpha}(\sigma) \neq 0$ and $(0.25)$ that

$$
\left|P\left(\frac{-1}{\sigma}\right)\right| \leq \underline{\lim }_{m \rightarrow \infty}\left\|P^{m}(S)[f(x)]\right\|_{L_{p, \alpha}}^{1 / m} .
$$

Since (0.26) holds for all $\sigma \in \operatorname{supp} \widetilde{f}_{\alpha}$, we obtain

$$
\sup \left\{\left|P\left(\frac{-1}{\sigma}\right)\right|: \sigma \in \operatorname{supp} \widetilde{f}_{\alpha}\right\} \leq \underline{\lim }_{m \rightarrow \infty}\left\|P^{m}(S)[f(x)]\right\|_{L_{p, \alpha}}^{1 / m} .
$$

Now, we will prove

$$
\sup \left\{\left|P\left(\frac{-1}{\sigma}\right)\right|: \sigma \in \operatorname{supp} \widetilde{f}_{\alpha}\right\} \geq \varlimsup_{\lim _{m \rightarrow \infty}}\left\|P^{m}(S)[f(x)]\right\|_{L_{p, \alpha}}^{1 / m}
$$

in the case where $f$ is a polynomial.

Indeed, put $M:=\operatorname{deg}(f)$. Hence,

$$
P^{m}(S)[f(x)]=\sum_{n=-M}^{M}\left(\delta_{n}\right)^{-1} P^{m}\left(\frac{-1}{n}\right) \widetilde{f}_{\alpha}(n) L_{n}^{\alpha}(x)(0<x<\infty)
$$

and

$$
\begin{gathered}
\left\|P^{m}(S)[f(x)]\right\|_{L_{p, \alpha}} \leq \sum_{n=-M}^{M}\left(\delta_{n}\right)^{-1} P^{m}\left(\frac{-1}{\sigma}\right) \widetilde{f}_{\alpha}(n)\left\|L_{n}^{\alpha}\right\|_{L_{p, \alpha}} \\
\leq\left[\sup \left\{\left|P\left(\frac{-1}{\sigma}\right)\right|: \quad n \in \operatorname{supp} \widetilde{f}_{\alpha}\right\}\right]^{m} \sum_{n=-M}^{M}\left(\delta_{n}\right)^{-1} \widetilde{f}_{\alpha}(n)\left\|L_{n}^{\alpha}\right\|_{L_{p, \alpha}}
\end{gathered}
$$

which gives

$$
\varlimsup_{\lim _{m \rightarrow \infty}}\left\|P^{m}(S)[f(x)]\right\|_{L_{p, \alpha}}^{1 / m} \leq \sup \left\{\left|P\left(\frac{-1}{\sigma}\right)\right|: \quad \sigma \in \operatorname{supp} \widetilde{f}_{\alpha}\right\} .
$$

That mean $(0.28)$ have been proved. From $(0.27)$ and $(0.28)$, it is easy to see

$$
\lim _{m \rightarrow \infty}\left\|P^{m}(S)[f(x)]\right\|_{L_{p, \alpha}}^{1 / m}=\sup \left\{\left|P\left(\frac{-1}{n}\right)\right|: n \in \operatorname{supp} \widetilde{f}_{\alpha}\right\}
$$

and the proof is complete.

Let $P(x)=x$, then we have the following result 
Corollary 0.4 Let $1 \leq p \leq \infty$ and let $f$ an arbitrary polynomial satisfying $\widetilde{f}_{\alpha}(0)=0$. Then, there exist the following limit

$$
\lim _{m \rightarrow \infty}\left\|S^{m}[f(x)]\right\|_{L_{p, \alpha}}^{1 / m}
$$

and

$$
\lim _{m \rightarrow \infty}\left\|S^{m}[f(x)]\right\|_{L_{p, \alpha}}^{1 / m}=\frac{1}{\operatorname{deg}_{1}(f)},
$$

where $\operatorname{deg}_{1}(f)=\inf \left\{n \in \mathbb{Z}_{+}: \widetilde{f_{\alpha}}(n) \neq 0\right\}$.

We denote by $T_{m}$ the set of the algebra polynomials of degree $\leq m$, and the error of approximation $E_{m}(f)$ of $f$ by elements from $T_{m}$ is

$$
E_{m}(f)=\inf _{P \in T_{m}}\|f-P\|_{L_{2, \alpha}} .
$$

Take it in mind, we have the following theorem

Theorem 0.5 Assume that $f(x) \in L_{2, \alpha}$. Then $L\{f(x)\} \in l_{2, \alpha}$ and

$$
\|f\|_{L_{2, \alpha}}=\sqrt{\Gamma(\alpha+1)}\|L\{f(x)\}\|_{l_{2, \alpha}} .
$$

Moreover,

$$
E_{m}(f)=\|f\|_{L_{2, \alpha}}-\sum_{n=0}^{m}\left(\widetilde{f_{\alpha}}(n)\right)^{2} \Gamma(\alpha+1)\left(\begin{array}{c}
n+\alpha \\
n
\end{array}\right) .
$$

Proof: We see that

$$
\begin{gathered}
\left\|f(x)-\sum_{n=0}^{m}\left(\delta_{n}\right)^{-1} c(n) L_{n}^{\alpha}(x)\right\|_{L_{2, \alpha}}=\int_{0}^{\infty} e^{-x} x^{\alpha}\left(f(x)-\sum_{n=0}^{m}\left(\delta_{n}\right)^{-1} c(n) L_{n}^{\alpha}(x)\right)^{2} d x \\
=\int_{0}^{\infty} e^{-x} x^{\alpha}(f(x))^{2} d x-2 \int_{0}^{\infty} e^{-x} x^{\alpha} f(x) \sum_{n=0}^{m}\left(\delta_{n}\right)^{-1} c(n) L_{n}^{\alpha}(x) d x \\
+\int_{0}^{\infty} e^{-x} x^{\alpha}\left(\sum_{n=0}^{m}\left(\delta_{n}\right)^{-1} c(n) L_{n}^{\alpha}(x)\right)^{2} d x .
\end{gathered}
$$

Note that

$$
\begin{gathered}
\int_{0}^{\infty} e^{-x} x^{\alpha} f(x) \sum_{n=0}^{m}\left(\delta_{n}\right)^{-1} c(n) L_{n}^{\alpha}(x) d x=\sum_{n=0}^{m} \int_{0}^{\infty} e^{-x} x^{\alpha} f(x)\left(\delta_{n}\right)^{-1} c(n) L_{n}^{\alpha}(x) d x \\
=\sum_{n=0}^{m}\left(\delta_{n}\right)^{-1} c(n) \widetilde{f}_{\alpha}(n) .
\end{gathered}
$$

Using (0.3) we obtain

$$
\begin{gathered}
\int_{0}^{\infty} e^{-x} x^{\alpha}\left(\sum_{n=0}^{m}\left(\delta_{n}\right)^{-1} c(n) L_{n}^{\alpha}(x)\right)^{2} d x=\sum_{1 \leq i, j \leq m} \int_{0}^{\infty} e^{-x} x^{\alpha}\left(\left(\delta_{n}\right)^{-2}(c(n))^{2} L_{i}^{\alpha}(x) L_{j}^{\alpha}(x)\right) d x \\
=\sum_{1 \leq i=j \leq m} \int_{0}^{\infty} e^{-x} x^{\alpha}\left(\left(\delta_{n}\right)^{-2}(c(n))^{2} L_{i}^{\alpha}(x) L_{j}^{\alpha}(x)\right) d x \\
=\sum_{n=0}^{m}\left(\delta_{n}\right)^{-1}(c(n))^{2} .
\end{gathered}
$$

Using (0.29), (0.32) and (0.34) we get

$$
\begin{aligned}
\left\|f(x)-\sum_{n=0}^{m}\left(\delta_{n}\right)^{-1} c(n) L_{n}^{\alpha}(x)\right\|_{L_{2, \alpha}} & =\|f\|_{L_{2, \alpha}}^{2}-2 \sum_{n=0}^{m}\left(\delta_{n}\right)^{-1} c(n) \widetilde{f}_{\alpha}(n)+\sum_{n=0}^{m}\left(\delta_{n}\right)^{-1}(c(n))^{2} \\
= & \|f\|_{L_{2, \alpha}}^{2}-\sum_{n=0}^{m}\left(\delta_{n}\right)^{-1}\left(\widetilde{f_{\alpha}}(n)\right)^{2}+\sum_{n=0}^{m}\left(\delta_{n}\right)^{-1}\left(\widetilde{f_{\alpha}}(n)-c(n)\right)^{2}
\end{aligned}
$$

which gives

$$
\inf _{\left(c_{0}, \ldots, c_{m}\right) \in \mathbb{R}^{m}}\left\|f(x)-\sum_{n=0}^{m}\left(\delta_{n}\right)^{-1} c(n) L_{n}^{\alpha}(x)\right\|_{L_{2, \alpha}}=\|f\|_{L_{2, \alpha}}^{2}-\sum_{n=0}^{m}\left(\delta_{n}\right)^{-1}\left(\widetilde{f}_{\alpha}(n)\right)^{2}
$$


Then, it follows from $\sum_{n=0}^{m}\left(\delta_{n}\right)^{-1} c(n) L_{n}^{\alpha}(x)$ which is the algebra polynomial of degree $m$

$$
E_{m}(f)=\|f\|_{L_{2, \alpha}}^{2}-\sum_{n=0}^{m}\left(\delta_{n}\right)^{-1}\left(\widetilde{f}_{\alpha}(n)\right)^{2}=\|f\|_{L_{2, \alpha}}-\sum_{n=0}^{m}\left(\widetilde{f}_{\alpha}(n)\right)^{2} \Gamma(\alpha+1)\left(\begin{array}{c}
n+\alpha \\
n
\end{array}\right) .
$$

Let $c(n)=\widetilde{f}_{\alpha}(n)$ for all $n=0,1, \ldots, m$, we obtain

$$
\left\|f(x)-\sum_{n=0}^{m}\left(\delta_{n}\right)^{-1} \widetilde{f}_{\alpha}(n) L_{n}^{\alpha}(x)\right\|_{L_{2, \alpha}}=\|f\|_{L_{2, \alpha}}^{2}-\sum_{n=0}^{m}\left(\delta_{n}\right)^{-1}\left(\widetilde{f}_{\alpha}(n)\right)^{2} .
$$

Therefore, since $\lim _{m \rightarrow \infty}\left\|f(x)-\sum_{n=0}^{m}\left(\delta_{n}\right)^{-1} \widetilde{f}_{\alpha}(n) L_{n}^{\alpha}(x)\right\|_{L_{2, \alpha}}=0$, we have $L\{f(x)\} \in l_{2, \alpha}$ and

$$
\|f\|_{L_{2, \alpha}}^{2}=\sum_{n=0}^{m}\left(\delta_{n}\right)^{-1}\left(\widetilde{f_{\alpha}}(n)\right)^{2}
$$

and then

$$
\sqrt{\Gamma(\alpha+1)}\|L\{f(x)\}\|_{L_{2, \alpha}}=\|f\|_{L_{2, \alpha}} .
$$

The proof is complete.

We are now able to present the following result

Theorem 0.6 Let $P(x)$ be the polynomial. Then for an arbitrary polynomial $f$, we always have

$$
\begin{aligned}
& \left\|P^{m}(R)[f(x)]\right\|_{L_{2, \alpha}} \leq \sup \left\{|P(-n)|: n \in \operatorname{supp} \widetilde{f}_{\alpha}\right\}\left\|P^{m-1}(R)[f(x)]\right\|_{L_{2, \alpha}}, \\
& \left\|P^{m}(R)[f(x)]\right\|_{L_{2, \alpha}} \geq \inf \left\{|P(-n)|: n \in \operatorname{supp} \widetilde{f}_{\alpha}\right\}\left\|P^{m-1}(R)[f(x)]\right\|_{L_{2, \alpha}}
\end{aligned}
$$

and

$$
\lim _{m \rightarrow \infty}\left\|P^{m}(R)[f(x)]\right\|_{L_{2, \alpha}}^{1 / m}=\operatorname{deg}(f) .
$$

Proof: We have known that

$$
L\left\{P^{m}(R)[f(x)]\right\}=P^{m}(-n) \widetilde{f}_{\alpha}(n) .
$$

Then it follows from Theorem 0.5 that

$$
\begin{gathered}
\left\|P^{m}(R)[f(x)]\right\|_{L_{2, \alpha}}=\sqrt{\Gamma(\alpha+1)}\left\|L\left\{P^{m}(R)[f(x)]\right\}\right\|_{l_{2, \alpha}} \\
=\sqrt{\Gamma(\alpha+1)}\left(\sum_{n=0}^{\infty}\left(P^{m}(-n) \widetilde{f}_{\alpha}(n)\right)^{2}\left(\begin{array}{c}
n+\alpha \\
n
\end{array}\right)\right)^{1 / 2} .
\end{gathered}
$$

Hence

$$
\begin{aligned}
\left\|P^{m}(R)[f(x)]\right\|_{L_{2, \alpha}}= & \sqrt{\Gamma(\alpha+1)}\left(\sum_{n \in \operatorname{Supp} \widetilde{f}_{\alpha}}\left(P^{m}(-n) \widetilde{f}_{\alpha}(n)\right)^{2}\left(\begin{array}{c}
n+\alpha \\
n
\end{array}\right)\right)^{1 / 2} \\
\leq & \sqrt{\Gamma(\alpha+1)} \sup \left\{|P(-n)|: n \in \operatorname{supp} \widetilde{f}_{\alpha}\right\} \\
& \times\left(\sum_{n \in \operatorname{supp} \widetilde{f}_{\alpha}}\left(P^{m-1}(-n) \widetilde{f}_{\alpha}(n)\right)^{2}\left(\begin{array}{c}
n+\alpha \\
n
\end{array}\right)\right)^{1 / 2} \\
= & \sup \left\{|P(-n)|: n \in \operatorname{supp} \widetilde{f}_{\alpha}\right\}\left\|P^{m-1}(R)[f(x)]\right\|_{L_{2, \alpha}}
\end{aligned}
$$


and (0.36) just been proved.

Now, we will prove (0.37). Indeed, we have

$$
\left\|P^{m}(R)[f(x)]\right\|_{L_{2, \alpha}}=\sqrt{\Gamma(\alpha+1)}\left(\sum_{n=0}^{\infty}\left(P^{m}(-n) \widetilde{f}_{\alpha}(n)\right)^{2}\left(\begin{array}{c}
n+\alpha \\
n
\end{array}\right)\right)^{1 / 2}
$$

which gives

$$
\left\|P^{m}(R)[f(x)]\right\|_{L_{2, \alpha}}=\sqrt{\Gamma(\alpha+1)}\left(\sum_{n \in \operatorname{Supp} \widetilde{f}_{\alpha}}\left(P^{m}(-n) \widetilde{f}_{\alpha}(n)\right)^{2}\left(\begin{array}{c}
n+\alpha \\
n
\end{array}\right)\right)^{1 / 2} .
$$

It follows that

$$
\begin{aligned}
\left\|P^{m}(R)[f(x)]\right\|_{L_{2, \alpha}} \geq & \sqrt{\Gamma(\alpha+1)} \inf \left\{|P(-n)|: n \in \operatorname{supp} \widetilde{f}_{\alpha}\right\} \\
& \times\left(\sum_{n \in \operatorname{supp} \widetilde{f}_{\alpha}}\left(P^{m-1}(-n) \widetilde{f}_{\alpha}(n)\right)^{2}\left(\begin{array}{c}
n+\alpha \\
n
\end{array}\right)\right)^{1 / 2} \\
= & \inf \left\{|P(-n)|: n \in \operatorname{supp} \widetilde{f}_{\alpha}\right\}\left\|P^{m-1}(R)[f(x)]\right\|_{L_{2, \alpha}},
\end{aligned}
$$

and $(0.37)$ just been proved. Note that, we check (0.37) from Theorem 0.1 . The proof is complete.

Since for all a polynomial $f$ and $n>\operatorname{deg}(f)$ then $\widetilde{f}_{\alpha}(n)=0$, we obtain $\operatorname{supp} \widetilde{f}_{\alpha} \subset\{0,1, \ldots \operatorname{deg}(f)\}$. Then, by applying Theorem 0.6 we have the following corollary

Corollary $0.7 \quad$ Let $P(x)$ be the polynomial. Then for an arbitrary polynomial $f$, we always have

$$
\begin{gathered}
\left\|P^{m}(R)[f(x)]\right\|_{L_{2, \alpha}} \leq \sup \{|P(-n)|: 0 \leq n \leq \operatorname{deg}(f)\}\left\|P^{m-1}(R)[f(x)]\right\|_{L_{2, \alpha},}, \\
\left\|P^{m}(R)[f(x)]\right\|_{L_{2, \alpha}} \geq \inf \{|P(-n)|: 0 \leq n \leq \operatorname{deg}(f)\}\left\|P^{m-1}(R)[f(x)]\right\|_{L_{2, \alpha}}
\end{gathered}
$$

and

$$
\lim _{m \rightarrow \infty}\left\|P^{m}(R)[f(x)]\right\|_{L_{2, \alpha}}^{1 / m}=\operatorname{deg}(f) .
$$

Let $P(x)=x$, then it follows from Theorem 0.6

Corollary $0.8 \quad$ Let $P(x)$ be the polynomial. Then for an arbitrary polynomial $f$, we always have

$$
\begin{gathered}
\left\|R^{m}[f(x)]\right\|_{L_{2, \alpha}} \leq \operatorname{deg}(f)\left\|P^{m-1}(R)[f(x)]\right\|_{L_{2, \alpha}}, \\
\left\|R^{m}[f(x)]\right\|_{L_{2, \alpha}} \geq \operatorname{deg}_{1}(f)\left\|P^{m-1}(R)[f(x)]\right\|_{L_{2, \alpha}},
\end{gathered}
$$

where $\operatorname{deg}_{1}(f)=\inf \left\{n \in \mathbb{Z}_{+}: \widetilde{f}_{\alpha}(n) \neq 0\right\}$ and

$$
\lim _{m \rightarrow \infty}\left\|R^{m}[f(x)]\right\|_{L_{2, \alpha}}^{1 / m}=\operatorname{deg}(f) .
$$

Remark. Since $f$ is the polynomial with degree $d, f(x)$ can express via

$$
f(x)=\sum_{n=0}^{d} a_{n} L_{\alpha}^{n}(x) .
$$

Then

$$
\left\{n \in \mathbb{Z}_{+}: \widetilde{f}_{\alpha}(n) \neq 0\right\}=\left\{n \in \mathbb{N}: a_{n} \neq 0\right\}
$$

and

$$
C=\inf \left\{|P(-n)|: n \in \mathbb{Z}_{+}, \widetilde{f_{\alpha}}(n) \neq 0\right\}=\inf \left\{|P(-n)|: n \in \mathbb{Z}_{+}, a_{n} \neq 0\right\} .
$$




\section{ACKNOWLEDGMENTS}

The work of the first author was supported by Portuguese funds through the CIDMA - Center for Research and Development in Mathematics and Applications, and the Portuguese Foundation for Science and Technology ("FCTFundação para a Ciência e a Tecnologia"), within project UID/MAT/ 0416/2013. The work of V.N.Huy was supported by Vietnam National University under grant number QG.16.08.

\section{REFERENCES}

[1] L. Debnath, On Laguerre Transforms, Bull Calcutta Math . Soc 55, 69-77 (1960).

[2] L. Debnath, Applications of Laguerre transform to the problem of oscillations of a very long and heavy chain, Annali dell' Univ. di Ferrara, Sezione VII-Scienze Mathematiche, IX, 149-151 (1961).

[3] L. Debnath, Application of Laguerre transform to heat conduction problem (1962), Annali dell' Univ. di Ferrara, Sezione VII-Scienze Mathematiche,X, 17-19 (1962).

[4] L. Debnath and D. Bhatta Integral transform and their applications, 2nd edition, Chapman \& Hall/CRC Press, Boca Raton, FL, 2007.

[5] V. Konyukh, B. G. Mikhailenko, and A. A. Mikhailov, Application of the integral Laguerre transforms for forward seismic modeling, J. Comp. Acous, 9(4), 1523-1541 (2001).

[6] J. McCully, The Laguerre transform, SIAM Review 2, 185-191 (1960).

[7] I. N. Sneddon, The use of Integral Transforms, McGraw-Hill, New York, 1972.

[8] J. C., Tranter Integral transform in mathematical physics, Champman and Hall, London, 1971. 